\title{
Continuous glucose monitoring: review of promising technologies
}

\author{
Monika Klimek $^{1, *}$, and Tytus Tulwin ${ }^{2}$ \\ ${ }^{1}$ Medical University of Lublin, Al. Racławickie 1, 20-059 Lublin, Poland \\ ${ }^{2}$ Lublin University of Technology, Department of Thermodynamics, Fluid Mechanics and Aircraft Propulsion, Nadbystrzycka 38D, 20- \\ 618 Lublin, Poland
}

\begin{abstract}
Despite the progress we have made in the management of diabetes it is still incurable and aggravating disease affecting all domains of quality of life. Uncontrolled diabetes associated with hyperglycemia leads to serious microvascular and macrovascular long-term complications. The proper longterm glycemic control is a key strategy for preventing the development or slowing the progression of diabetes complications, thus there is a crucial role of new technologies in the diabetes care. New technologies in diabetology are developing dynamically in recent years and therefore this is a topical issue. In this paper we describe current and developing continuous glucose monitoring technologies and their usefulness in promoting optimal glycemic control, influence on personalized diabetes managements and the functioning of patients. Moreover we review knowledge about flash glucose monitoring and close-loop system. This review examines studies published before 31st August 2018.
\end{abstract}

\section{Introduction}

\subsection{Diabetes mellitus - pathophysiology}

Diabetes mellitus (DM) is a chronic disease that results from insufficient insulin secretion or tissue insulin resistance. Insulin is a hypoglycemic hormone that plays a fundamental role in regulating glucose metabolism by decreasing blood glucose concentration and maintaining normal glycemic. Regardless of the type of diabetes, a common effect of the uncontrolled disease is hyperglycemia which leads to serious microvascular or macrovascular complications over time such as cardiovascular disease, blindness, neuropathy and nephropathy that are mostly irreversible (1).

\subsection{Diabetes mellitus - epidemiology}

In the 21 st century, diabetes has become a global epidemic. According to the most recent IDF (International Diabetes Federation) report for the year 2017, the incidence of diabetes has been increasing at a rate much higher than predicted and the total population of diabetes patients amounts approximately 425 million individuals, equalling to $8,8 \%$ of the population aged 20-79 years (2). The results of the largest epidemiological studies conducted in Poland in recent years estimate diabetes prevalence at around $6,7-9,8 \%(3)$.

\subsection{History of glucose measurement methods}

DM has been known for almost two thousand years since the Greek physician Aretaeus described classical symptoms of disease (constant thirst, excessive urination, loss of weight) and used the term "diabetes" for the first time in $150 \mathrm{AD}$ (4). The pathophysiology of DM remained unknown, and it was an incurable disease - patients have died within a few years after the diagnosis of the disease. Only after further discoveries such as the discovery the sweetness of urine (Thomas Willis, 1674), an identification the causes of the sweet taste of urine (Matthew Dobson, 1775) and the discovery that the amount of sugar excreted in urine depends on the type of food (John Rollo, 1797), the 19th century brought breakthrough discoveries concerning the pathophysiology of DM - the discovery of the islet cells of the pancreas (Paul Langerhans, 1869) and the discovery of a role for the pancreas in diabetes (Naunyn, Minkowski and Mering, 1889). These findings enabled the revolution in diabetes therapy - the discovery of insulin by Banting, Best and Collip, which was one of the most important events in the history of medicine. The first injection of a crude beef pancreatic extract was given to 14-year-old Leonard Thompson on January 11, 1922. The discovery of insulin was awarded the Nobel Prize in Medicine in 1923 (5).

Diabetes management requires adequate insulin dosing depending on diet, physical activities and many other factors affecting blood glycemia. Urine tests were the first method allowing controlling the glucose level. Later urine test strips have been used, but both had poor results in managing blood glucose level and led to early severe vascular complications (6).

The first blood glucose test strip which was a more reliable diagnostic tool was developed in 1965. Although the visually monitored blood glucose test strips were widely used, disadvantages such as color fading and highly significant visual variations in the assessment of colors across the range of glucose concentrations stimulated the development of more accurate electronic glucose meters $(7,8)$.

Self-monitoring of blood glucose has become possible since the first portable glucose meters were created in the late 1960s and as Ames Reflectance Metering became available 1970. Self-monitoring blood glucose (SMBG) meters are devices that measure blood glucose concentration on a drop of capillary blood by means of glucose-oxidase reaction. The SMBG meter using fingerstick blood samples, test strips, and portable meters is easy and fast. The technology improvement have reduced the

* Corresponding author: monika.klimekk@gmail.com 
meters and reagent strips costs and SMBG has become the most widely used method for measuring blood glucose levels and an essential tool in diabetes self-managing. SMBG improved diabetes management by enabling patients to monitor their blood glucose levels and become partners along with health professionals which significantly improved glycemic control among diabetics (8).

Enormous progress has been made in glucose metres over the past 4 decades. The number of smaller and more accurate SMBG continued to increase. Many metres now have more advanced data-handling capabilities and additional features to record daily insulin dose history, carbohydrate intake and exercise. Despite the undeniable advantages, the limitations of this method must not be forgotten. Pain associated with the finger prick is frightening for some patient and it might be an unpleasant experience makes patients less likely to control blood glucose levels often. From the health professionals' point of view the main weakness of this method is insufficient sampling frequency which provides the current capillary blood glucose concentration without information about the glucose trend - intermittent capillary glucose testing with three to four finger-stick tests providing a snapshot of dayto-day glucose control. In contrast, glycated haemoglobin (HbAlc) reflects average blood glucose level during the preceding 120 days and it has gain high acceptance as an index for assessing the risk of complications and as a prognosis indicator for the success of treatment of diabetes. Nevertheless, none of these methods provides information about major changes in glucose level such as hypoglycemia and hyperglycemia during a day. Better long-term diabetes control resulting in a reduction in $\mathrm{HbAlc}$ is associated with decreases complications. However, tight glycemia control is associated with the risk of hypoglycemia remains the most feared complication of insulin therapy since it was described, which limits the possibility of intensification of treatment (8-10).

\subsection{Purpose}

This paper focuses on describing current and developing continuous glucose monitoring technologies used in the management of patients with diabetes. We describe current technologies and their usefulness in promoting optimal glycemic control, influence on personalized diabetes managements and the functioning of patients. We also review available knowledge about flash glucose monitoring and close-loop system.

To the best of our knowledge it is the most recent comprehensive review of continuous glucose monitoring and closed loop system technologies, but we clarify at the outset that our review does not completely exhaust the topic which is related to the large amount of research conducted on this subject and the rapid introduction of new devices to the market.

\section{Material and methods}

The review combines the findings of literature searching in computerised databases and manual searches. We searched for studies published before 31st August 2018, on PubMed, Google Scholar and product websites. To ensure a broad search, we used the keywords 'diabetes'
AND 'continuous glucose monitoring' OR 'flash glucose monitoring' OR ‘closed loop'.

\section{Results}

\subsection{Continuous Glucose Monitoring}

The introduction of continuous glucose monitoring (CGM) that measures glucose levels continuously and provides real-time results is another breakthrough in diabetes care. Typically CGM comprise glucose-sensing device inserted in the abdomen or on the arm, electrochemically measuring glucose levels in subcutaneous tissues every $1-5 \mathrm{~min}$ and providing information about interstitial glucose, a monitor to display the information, and a transmitter that transmits the sensor data to the monitor. Moreover CGM-based treatment almost eliminates the need of finger-sticks - it requires finger-sticks just for a calibration procedure, usually only twice a day.

The first successfully commercialized CGM sensor products got FDA approval in 1999 (The Guardian ${ }^{\circledR}$ RT system, Medtronic Minimed, Northridge, CA) (9). Early models of CGM were readable only by a physician (professional CGM) and provided a retrospectively summary of glycaemic control. Currently available CGM devices offer the real-time monitoring, provide graphs of glucose trends and alerts to impending hypoand hyperglycemia events that may be used for diabetic self-management.

Clinical benefits of real-time measurements of glucose levels using CGM have been widely confirmed in metanalysis in type 1 diabetes (T1D). CGM allows insulin dose modification and reduced exposure to hypoglycaemia suggesting significant benefits for average glucose control. The regular CGM use is associated with a lower level of $\mathrm{HbAlc}$ and lower risk of hypoglycemia in T1D (10-14). Most studies using CGM have been done in patients with T1DM while there are only limited studies evaluating the effectiveness of CGM usage in type 2 diabetes (T2D). The vast majority of diabetics are $\mathrm{T} 2 \mathrm{D}$, thereby the number of insulin users requiring frequent glucose monitoring is mostly $\mathrm{T} 2 \mathrm{D}$ as well. Moreover it's known that less than a fifth of T2D patients reach target $\mathrm{HbA} 1 \mathrm{c}$, so it's clear that new more efficient and user-friendly monitoring and treatment methods are needed improve glycemic control in that patients (15).

Effects of continuous glucose monitoring on glycemia and HbA1c reduction for T2D management has been summarized in Vigersky and others. The analysis containing trials till 2016 showed applicability of CGM in T2D. Professional CGM often referred as a Holter monitor for glucose measurements has been demonstrated as effective in $\mathrm{HbA} 1 \mathrm{C}$ improving and as an educational tool in patients with T2D. Evidence for use of personal real-time CGM are limited, but the evidence suggests its effectiveness in improving glycemic control in T2D patients (16). Recent narrative review of 11 studies (2001-2017) provide evidence of that promotes improvements in glycemic, weight control and behaviour change in T2D adult patients (17). 
Subcutaneous CGM may be useful in critically ill patients with insulin treatment. It has been shown that it's as safe and effective as intermittent measurements using blood samples and it enables to detect minimize changes in glucose levels warning early about hypo- and hyperglycemia. Moreover it may reduce nursing workload and daily costs of glycemic control (18).

Making therapeutic decisions and insulin dosing based on blood glucose readings required accurate measurements. The acceptable level of accuracy criteria for SMBG meters are defined as within $15 \%$ of a reference plasma value for blood glucose above 100 $\mathrm{mg} / \mathrm{dL}$ or within $15 \mathrm{mg} / \mathrm{dL}$ for blood glucose below 100 $\mathrm{mg} / \mathrm{dL}$. With the sensor accuracy improving the quality of the data coming from CGM devices and therefore the CGM usability has been rapidly improving over the past few years. The average accuracy for devices is indicated by the mean absolute relative difference (MARD). SMBG systems using finger-stick blood samples have a MARD between $5 \%$ and $10 \%$ (the mean error may be 5$10 \mathrm{mg} / \mathrm{dl}$ ). Historically, CGM devices had lower measurement accuracy compared to standard SMBG so the glucose value had to be verified with traditional SMBG before taking insulin or adjusting other components of therapy. Accuracy of the first successfully commercialized product was $15.8 \%$. Thus, a great attention has been given to improving accuracy, what has resulted in greater accuracies demonstrated in current commercially available CGM devices. MARD for currently commercially available CGM devices have recently been analysed and equals from $9 \%$ to $14,5 \%$, thus the accuracy of some current CGM systems is now at levels comparable to SMBG (19). Nevertheless, patients using CGM systems are still encouraged to confirm their CGM result with a finger-stick test before making management decisions or to verify the result in case of hypo- and hyperglycemia and symptoms do not match the device's reading. Increased accuracy, especially at dangerous and life-threatening low blood glucose levels, and reduced calibration requirement remains a technical challenge $(20,21)$. In Table 1 we summarize the accuracy of available CGM devices.

Dexcom launched the G4 Platinum CGM sensor in 2012 (MARD 13\%), after algorithmic changes in 2014 sensor has gained better accuracy (MARD 9\%) $(22,23)$. The Dexcom G5 Mobile was the first CGM system approved for adults and pediatric patients two years of age and older (23). The sixth-generation factorycalibrated real-time CGM system (Dexcom G6) requires only one finger-stick calibration per day has a longer sensor life than the G5 (10 days vs. 7 days) and its MARD equals 9\%. FDA approved G6 CGM in March 2018 and it's available from June 2018 (24-26). Medtronic launched the Enlite Sensor in 2011 (MARD $13.6 \%$ ) and Guardian Sensor 3 five years later (MARD $10.6 \%$ in the abdomen, $9.1 \%$ in the $\operatorname{arm})(27,28)$. Guardian Sensor 3 has been recently approved by FDA to be used in the Minimed $670 \mathrm{G}$ system hybrid closed loop system (29). Abbott Company launched the FreeStyle Navigator II CGM system in 2011 (MARD $14,2-14,5 \%)(30)$. While most CGM systems have a lifetime of 6-14 days, small implantable CGM Eversense Sensor System marketed by Senseonics Company lasts up to 6 months. The sensor needs to be inserted and removed in a doctor's office during a minor surgical procedure - replacement is needed every 90-180 days. Transmitter device adhered to the skin over the implanted sensor that sends data to a smartphone app. This system still requires two finger-stick calibrations per day, and its MARD equal 8.8\% (31-33).

Despite of advantages of using CGM, they are still uncommon. The distribution of CGM sensors is limited to mainly T1D but the most recent data suggest that only $17-30 \%$ of T1D patients use CGM. Using CGM is even less common among T2D patients. The greatest barrier to CGM widespread use is the high ongoing cost related to high device costs and lack of insurance coverage which is also the most common reason for stopping CGM use $(14,34,35)$.

Table 1. Summary of mean absolute relative difference (MARD) of CGM devices. ${ }^{1}$ Closed loop system; ${ }^{2}$ Flash glucose monitoring system.

\begin{tabular}{|c|c|c|c|c|}
\hline Company & Sensor system & FDA approval & Accuracy (MARD) & References \\
\hline \multirow{4}{*}{ Dexcom } & \multirow{2}{*}{ G4 Platinum } & 2012 & $13 \%$ & $(22)$ \\
\hline & & 2014 & $9 \%$ & (23) \\
\hline & G5 Mobile & 2016 & $9 \%$ & $(23,36)$ \\
\hline & G6 & 2018 & $9 \%$ & $(24-26)$ \\
\hline \multirow{3}{*}{ Medtronic } & Enlite Sensor & 2011 & $13.6 \%$ & (27) \\
\hline & Guardian Sensor 3 & 2016 & $9.6 \%$ & $(28)$ \\
\hline & MiniMed 670G ${ }^{1}$ & $\begin{array}{c}2016 \text { (14 years and older), } \\
2018 \text { (under } 7 \text { years) }\end{array}$ & Integrated with Guardian Sensor 3 & (29) \\
\hline \multirow{3}{*}{ Abbott } & Navigator II & 2011 & $14.1 \%$ & $(30)$ \\
\hline & \multirow{2}{*}{ FreeStyle Libre ${ }^{2}$} & 2017 & $9.7 \%$ & $(37,38)$ \\
\hline & & 2018 & $9.4 \%$ & $(38,39)$ \\
\hline \multirow{2}{*}{ Senseonics } & \multirow{2}{*}{ Eversense } & $\mathrm{x}$ & $11.6 \%$ & $(31)$ \\
\hline & & 2018 & $8.8 \%$ & $(32,33)$ \\
\hline
\end{tabular}




\subsection{Flash Glucose Monitoring}

Extension of the glucose monitoring sensors wearing time and its accuracy were the most meaningful challenges in past years. Flash glucose monitoring system (FGM) is the unique and newest method of glucose testing based on the measurement of glucose concentrations in the interstitial fluid without calibration requirements. In contrast to the available CGM which still require calibration using capillary blood glucose readings, the FGM system is factory calibrated, and it does not require calibration by the user. Finger-stick testing is not required to confirm the data's accuracy so users can dose insulin based on its readings. Finger-stick checks are needed only in some situations when glucose levels are rapidly changing, or when symptoms don't match the system's readings. FGM is seen as a hybrid between standard glucometers and continuous glucose monitoring systems. Reduced cost and factory calibration are two of the most innovative features of FGM. Flash glucose monitoring sensor is inserted on a patient's upper arm. The scannable sensor transmits an instantaneous glucose level and trend graph to the separate reader device when it is swiped close to the sensor. FGM requires the patient to place the receiver close to the sensor for data visualization, thus permanent connection to an external device isn't possible. It does not provide continuous glucose monitoring, trend data in real time, and does not have hypo- or hyperglycemia alarms for real-time blood sugars. The glucose level in unknown when it's not mannually tested, e.g. during the night, however the sensor can be scanned by someone else whiles patient is asleep $(1,31)(1,32)$.

Flash glucose monitoring systems is actually provided by the only one company, Abbott Diabetes Care. Their FGM device - FreeStyle Libre was introduced in 2014 and it had received FDA approval in September 2017. The first approved product could be worn for 10 days (MARD 9,7\%), the second-generation of the FreeStyle Libre Flash (MARD $9,4 \%$ ) that can be worn for 14-days has been recently approved (37-39).

FGM in type 2 diabetes with intensive insulin therapy can be a replacement for SMBG. It's not clear if FGM may improve glycemic control more than CGM or SMBG, but it's proved that higher scanning frequency is associated with better glycemic measures including lower HbA1c $(21,41,42)$.

\subsection{Closed-loop}

CGM is one of the pillars of closed-loop control systems (the so-called artificial pancreas) for automatic delivery of insulin and glucagon. Rather than waiting until the observed glucose has fallen below a specified threshold glucose level, giving an alarm and relying on the patient or a family member to take the necessary action, real-time CGM can suspend insulin delivery from an insulin pump.

The Medtronic MiniMed 670G System is the first FDA approved hybrid closed loop system that monitors glucose and automatically adjusts the delivery of long acting or basal insulin based on the user's glucose reading consists of a continuous glucose monitor (CGM) that measures the user's glucose levels for up to seven days, an insulin pump that delivers insulin to the user, and a glucose meter used to calibrate the CGM. This device was approved on September
28, 2016 for users aged 14 years and older, and has been recently approved in users under seven years of age (29)

Current limited results suggest that the usage of a fully automated closed-loop insulin delivery system in patients with diabetes in the general ward is safe and effective as well as standard subcutaneous insulin therapy. The glucose management is significantly improved which is shown as reductions in $\mathrm{HbA1c}$, hyperglycemia and hypoglycemia (4347).

\section{Discussion}

Despite the discovery of insulin and then hypoglycaemic drugs which prolong the life expectancy of patients with DM, it's still remains an incurable disease and a major cause of death and health complications throughout the world. Statistically, visual acuity occurs after 5 years of diabetes, renal failure after 15 years, cardiovascular diseases after 30 years. As the proper long-term glycemic control is a key strategy for preventing the development or slowing the progression of diabetes complications, diabetes management remains a serious technology challenge.

New technologies such as continuous blood glucose monitoring devices, insulin pumps, computerised mathematical algorithms, and closed-loop artificial pancreas systems have been developed to improve blood glucose control, prevent long-term complications, and improve patient quality of life as well. The approaches offer promising glucose control to people with diabetes, however we still have a long journey towards a cure.

\section{References}

1. R. Donnelly, A. M. Emslie-Smith, I. D. Gardner, and A. D. Morris, BMJ 320 (7241), 1062-1066 (2000)

2. International Diabetes Federation. IDF Diabetes Atlas, 8th edn. Brussels (Belgium: International Diabetes Federation, 2017), http://www.diabetesatlas.org

3. M. Klimek, sJ. Knap, T. Tulwin, M. Trojnar, and G. Dzida, Clin. Diabetol. 7 (3), 145-150 (2018)

4. F. Henschen, Med. Hist. 13 (2), 190-192 (1969)

5. C. Stylianou and C. Kelnar, J. R. Soc. Med. 102 (7), 298-303 (2009)

6. N. Moodley, U. Ngxamngxa, M. J. Turzyniecka, and T. S. Pillay, J. Clin. Pathol. 68 (4), 258-264 (2015)

7. R. D. Cheeley and S. M. Joyce, Am. J. Emerg. Med. 8 (1), 11-15 (1990)

8. S. F. Clarke and J. R. Foster, Br. J. Biomed. Sci. 69 (2), 83-93 (2012)

9. L. A. Scrimgeour, B. A. Potz, F. W. Sellke, and M. R. Abid, Clin. Med. Res. 6 (6), 173-176 (2017)

10. R. A. Ajjan, Diabetes Technol. Ther. 19 (Suppl 2), S27-S-36 (2017)

11. K. Benkhadra, F. Alahdab, S. Tamhane, Z. Wang, L. J. Prokop, I. B. Hirsch, D. Raccah, J.-P. Riveline, O. Kordonouri, and M. H. Murad, Clin. Endocrinol. (Oxf.) 86 (3), 354-360 (2017)

12. R. W. Beck, T. Riddlesworth, K. Ruedy, A. Ahmann, R. Bergenstal, S. Haller, C. Kollman, D. Kruger, J. B. McGill, W. Polonsky, E. Toschi, H. Wolpert, D. Price, 
and DIAMOND Study Group, JAMA 317 (4), 371378 (2017)

13. R. A. Ajjan, K. Abougila, S. Bellary, A. Collier, B. Franke, E. B. Jude, G. Rayman, A. Robinson, and B. M. Singh, Diab. Vasc. Dis. Res. 13 (3), 211-219 (2016)

14. Ont. Health Technol. Assess. Ser. 18 (2), 1-160 (2018)

15. J. M. Polinski, J. G. Connolly, B. H. Curtis, J. D. Seeger, K. Gaskins, M. Perez, B. F. Smith, and W. H. Shrank, Prim. Care Diabetes 8 (2), 101-109 (2014)

16. R. Vigersky and M. Shrivastav, J. Diabetes Complications 31 (1), 280-287 (2017)

17. P. J. Taylor, C. H. Thompson, and G. D. Brinkworth, J. Diabetes Investig. 9 (4), 713-725 (2018)

18. S. C. J. van Steen, S. Rijkenberg, J. Limpens, P. H. J. van der Voort, J. Hermanides, and J. H. DeVries, Sensors 17 (1), (2017)

19. G. Cappon, G. Acciaroli, M. Vettoretti, A. Facchinetti and G. Sparacino, Electronics 6 (3), 65 (2017)

20. J. Pettus and S. V. Edelman, J. Diabetes Sci. Technol. 11 (1), 138-147 (2017)

21. D. Praktyczna, Diabetol. Prakt. 4 (1), 1-94 (2018)

22. M. Christiansen, T. Bailey, E. Watkins, D. Liljenquist, D. Price, K. Nakamura, R. Boock, and T. Peyser, Diabetes Technol. Ther. 15 (10), 881-888 (2013)

23. T. S. Bailey, A. Chang, and M. Christiansen, J. Diabetes Sci. Technol. 9 (2), 209-214 (2015)

24. V. N. Shah, L. M. Laffel, R. P. Wadwa, and S. K. Garg, Diabetes Technol. Ther. 20 (6), 428-433 (2018)

25. U.S. Food and Drug Administration. FDA authorizes first fully interoperable continuous glucose monitoring system, streamlines review pathway for similar devices.

2018. https://www.fda.gov/NewsEvents/Newsroom/PressAn nouncements/ucm602870.htm

26. Dexcom G6 CGM System User Guide, 2018. https://s3-us-west-2.amazonaws.com/dexcompdf/G6CGM-Users-

Guide.pdf?.ga=2.240766279.578548036.1539290424 $-1566612695.1539121628$

27. T. S. Bailey, A. Ahmann, R. Brazg, M. Christiansen, S. Garg, E. Watkins, J. B. Welsh, and S. W. Lee, Diabetes Technol. Ther. 16 (5), 277-283 (2014)

28. M. P. Christiansen, S. K. Garg, R. Brazg, B. W. Bode, T. S. Bailey, R. H. Slover, A. Sullivan, S. Huang, J. Shin, S. W. Lee, and F. R. Kaufman, Diabetes Technol. Ther. 19 (8), 446-456 (2017)

29. U.S. Food and Drug Administration. RecentlyApproved Devices - Minimed 670G System. https://www.fda.gov/MedicalDevices/ProductsandMe dicalProcedures/DeviceApprovalsandClearances/Rece ntly-ApprovedDevices/ucm614616.htm

30. M. Geoffrey, R. Brazg, and W. Richard, J. Diabetes Sci. Technol. 5 (1), 99-106 (2011)

31. J. Kropff, P. Choudhary, S. Neupane, K. Barnard, S. C. Bain, C. Kapitza, T. Forst, M. Link, A. Dehennis, and J. H. DeVries, Diabetes Care 40 (1), 63-68 (2017)

32. M. P. Christiansen, L. J. Klaff, R. Brazg, A. R. Chang, C. J. Levy, D. Lam, D. S. Denham, G. Atiee, B. W. Bode, S. J. Walters, L. Kelley, and T. S. Bailey, Diabetes Technol. Ther. 20 (3), 197-206 (2018)
33. U.S. Food and Drug Administration. RecentlyApproved Devices - Eversense Continuous Glucose Montioring System. https://www.fda.gov/MedicalDevices/ProductsandMe dicalProcedures/DeviceApprovalsandClearances/Rece ntly-ApprovedDevices/ucm614564.htm

34. M. Vettoretti, G. Cappon, G. Acciaroli, A. Facchinetti, and G. Sparacino, J. Diabetes Sci. Technol. 12 (5), 1064-1071 (2018)

35. N. C. Foster, K. Miller, L. Dimeglio, D. M. Maahs, W. V. Tamborlane, R. M. Bergenstal, M. A. Clements, M. R. Rickels, E. Smith, B. A. Olson, and R. Beck, Diabetes 67 (Supplement 1), 1689-P (2018)

36. U.S. Food and Drug Administration. Premarket Approval - Dexcom G5 Mobile Continuous Glucose Monitoring System. https://www.accessdata.fda.gov/scripts/cdrh/cfdocs/cf $\mathrm{pma} / \mathrm{pma} . \mathrm{cfm}$ ?id=P120005S041

37. T. Bailey, B. W. Bode, M. P. Christiansen, L. J. Klaff, and S. Alva, Diabetes Technol. Ther. 17 (11), 787-794 (2015)

38. U.S. Food and Drug Administration. Summary of safety and effectiveness data - FreeStyle Libre Flash Glucose Monitoring System. https://www.accessdata.fda.gov/cdrh_docs/pdf16/P16 0030B.pdf

39. U.S. Food and Drug Administration. RecentlyApproved Devices - Freestyle Libre 14 Day Flash Glucose Monitoring System. https://www.fda.gov/MedicalDevices/ProductsandMe dicalProcedures/DeviceApprovalsandClearances/Rece ntly-ApprovedDevices/ucm614903.htm

40. L. Heinemann and G. Freckmann, J. Diabetes Sci. Technol. 9 (5), 947-950 (2015)

41. T. C. Dunn, Y. Xu, G. Hayter, and R. A. Ajjan, Diabetes Res. Clin. Pract. 137, 37-46 (2018)

42. T. Haak, H. Hanaire, R. Ajjan, N. Hermanns, J.-P. Riveline, and G. Rayman, Diabetes Ther. 8 (1), 55-73 (2017)

43. H. Thabit, L. Leelarathna, M. E. Wilinska, D. Elleri, J. M. Allen, A. Lubina-Solomon, E. Walkinshaw, M. Stadler, P. Choudhary, J. K. Mader, S. Dellweg, C. Benesch, T. R. Pieber, S. Arnolds, S. R. Heller, S. A. Amiel, D. Dunger, M. L. Evans, and R. Hovorka, Diabetes Technol. Ther. 17 (11), 801-807 (2015)

44. L. Bally, H. Thabit, S. Hartnell, E. Andereggen, Y. Ruan, M. E. Wilinska, M. L. Evans, M. M. Wertli, A. P. Coll, C. Stettler, and R. Hovorka, N Engl J Med. 379 (6), 547-556 (2018)

45. D. Rodbard, Diabetes Technol. Ther. 19 (Suppl 3), S25-S-37 (2017)

46. R. M. Bergenstal, S. Garg, S. A. Weinzimer, B. A. Buckingham, B. W. Bode, W. V. Tamborlane, and F. R. Kaufman, JAMA 316 (13), 1407-1408 (2016)

47. S. K. Garg, S. A. Weinzimer, W. V. Tamborlane, B. A. Buckingham, B. W. Bode, T. S. Bailey, R. L. Brazg, J. Ilany, R. H. Slover, S. M. Anderson, R. M. Bergenstal, B. Grosman, A. Roy, T. L. Cordero, J. Shin, S. W. Lee, and F. R. Kaufman, Diabetes Technol. Ther. 19 (3), 155-163 (2017) 